\title{
ROLE OF LOCAL HEMOSTATIC AGENTS AS ADJUVANT IN CONTROL OF PRIMARY POSTPARTUM HEMORRHAGE
}

\author{
By \\ Mohamed El-Saghier El-Hofey, Hossam Al-Din Hussein Kamel and Adel \\ Aly El-Boghdady \\ Department of Obstetrics and Gynecology, Faculty of Medicine, Al-Azhar University \\ Corresponding author: Mohamed El-Saghier El-Hofey, \\ E-mail: mohamedelhofey007@gmail.com
}

\begin{abstract}
Background: Hemostasis is an integral and very important aspect of surgical practice. In the developing world, about $1.2 \%$ of deliveries are associated with PPH and when PPH occurred about 3\% of women died. Causes of postpartum hemorrhage are uterine atony, trauma, retained placenta or placental abnormalities, and coagulopathy, commonly referred to as the "four Ts".
\end{abstract}

Objective: To study the effect of gelfoam in hemostasis as adjuvant in control of primary postpartum hemorrhage.

Patients and Methods: This was a Cohort study conducted at Sayed Galal University Hospital, Al-Azhar University Faculty of Medicine during the period from May 2019 to May 2020. A number of 60 cases of postpartum hemorrhage and role of gel foam in hemostasis as adjuvant in control of primary postpartum hemorrhage.

Results: The findings of the present study indicate that local hemostatic agent "gel foam" was useful as adjuvant in control of primary postpartum hemorrhage in cesarean section. Local hemostats reduce the use of systemic hemostats, shorten the time required to achieve hemostasis. The delay in the recovery of bowel motility was due to the local hypersensitivity reaction caused by gelatin sponge and/or dislocation of this local hemostat.

Conclusion: Local hemostatic agent "gel foam" was useful in adjuvant in control of primary postpartum hemorrhage in cesarean section.

Keywords: Active management of the third stage of labor, postpartum hemorrhage, glutaraldehyde and bovine albumin adhesive.

\section{INTRODUCTION}

Primary postpartum bleeding is defined as blood loss in excess of $500 \mathrm{ml}$ following vaginal delivery or $1000 \mathrm{ml}$ following caesarean section in the first 24 hours following birth. Secondary postpartum bleeding is that which occurs after the first day and up to six weeks after childbirth (Weeks, 2015).
In the developing world about $1.2 \%$ of deliveries are associated with PPH and when PPH occurred about 3\% of women died (Ononge et al., 2016).

Globally, it occurs about 8.7 million times and results in 44,000 to 86,000 deaths per year making it the leading cause of death during pregnancy. About 0.4 women per 100,000 deliveries die 
from PPH in the United Kingdom while about 150 women per 100,000 deliveries die in sub-Saharan Africa. Rates of death have decreased substantially since at least the late 1800s in the United Kingdom. In an analysis of population-based data from the United States National Inpatient Sample, the incidence was between 2 and 3 percent during the years 1994 to 2006 and 3 percent in 2012 to 2013. GBD 2013 Mortality and Causes of Death, Collaborators (Abubakar et al., 2015).

Risk factors are abnormalities of uterine contraction, overdistension of uterus, polyhydramnios, multiple gestation, macrosomia, uterine muscle exhaustion, rapid labour, prolonged labour, high parity, oxytocin use, intraamniotic infection, fever, prolonged rupture of membranes, Functional/anatomic distortion of uterus, uterine-relaxing medications, bladder distension, which may prevent uterine contraction, fibroids, placenta previa, uterine anomalies, halogenated anesthetics nitroglycerin history of previous $\mathrm{PPH}$, history of hereditary coagulopathies or liver disease acquired in pregnancy, idiopathic thrombocytopenic purpura, thrombocytopenia with preeclampsia, disseminated intravascular coagulation gestational, hypertensive disorder of pregnancy with adverse conditions a) Dead fetus in utero b) Severe infection c) Abruption d) amniotic fluid embolus Bruising and antepartum hemorrhage Sudden collapse (Wormer and Brynat, 2018).

Causes of postpartum hemorrhage are uterine atony, trauma, retained placenta or placental abnormalities, and coagulopathy, commonly referred to as the "four Ts" (Sebghati and Chandraharan, 2017).

Gelfoam Sterile Compressed Sponge is a water-insoluble, hemostatic device prepared from purified porcine skin gelatin, and capable of absorbing up to 45 times its weight of whole blood. The absorptive capacity of Gelfoam is a function of its physical size, increasing as the size of the gelatin sponge increases (Behrens et al., 2014).

The present work aimed to study the effect of gelfoam in hemostasis as adjuvant in control of primary postpartum hemorrhage.

\section{PATIENTS AND METHODS}

This was a Cohort study conducted at Sayed Galal University Hosptial, AlAzhar University Faculty of Medicine during the period from May 2019 to May 2020.

Study population: A number of 60 cases of postpartum hemorrhage and role of gel foam in hemostasis as adjuvant in control of primary postpartum hemorrhage

Inclusion criteria: Age (25-39). Parity (2 - 4). Gravity (3 - 5).

Exclusion criteria: No maternal disease during pregnancy, no maternal heart diseases, no maternal hypertension and no maternal blood disease during pregnancy. Maternal autoimmune disease. Hepatitis C or hepatitis B disease. Blood disease.

Protocols to manage postpartum hemorrhage were recommended to ensure the rapid giving of blood products when needed. A detailed stepwise management protocol has been introduced by the California Maternity Quality Care Collaborative. It described 4 stages of 
obstetrical hemorrhage after childbirth and its application reduced maternal mortality.

Stage 0: Normal - treated with fundal massage and oxytocin. Stage 1: More than normal bleeding - establish largebore intravenous access, assemble personnel, increase ox, consider use of methergine, perform fundal massage, prepare 2 units of packed red blood cells. Stage 2: Bleeding continues - check coagulation status, assemble response team, move to operating room, place intrauterine balloon, administer additional uterotonics carboprosttromethamine), consider: uterine artery embolization, GelFoam, dilatation and curettage, and laparotomy with uterine compression stitches or hysterectomy. Stage 3: Bleeding continues - activate massive transfusion protocol, mobilize additional personnel, recheck laboratory tests, perform laparotomy, consider hysterectomy.

Ethics and Patient Consent: All procedures followed Al Azhar University Ethical Committee regulations. Oral consent was taken from all patients before collecting any information or starting any procedure.

\section{Statistical Analysis:}

Data were collected, revised, coded and entered to the Statistical Package for the Social Sciences (IBM SPSS) version 23. The quantitative data were presented as mean, standard deviations, ranges, median and interquartile range (IQR) when their distribution found parametric. Also qualitative variables were presented as number and percentages. The comparison between the studied groups regarding qualitative data was done by using Chi-square test. The comparison between two independent groups with quantitative data and parametric distribution were done by using Independent t-test while two independent groups with quantitative data and nonparametric distribution were done by using Mann-Whitney test. The confidence interval was set to $95 \%$ and the margin of error accepted was set to $5 \%$. So, the pvalue was considered significant when $\mathrm{P} \leq$ 0.05 .

\section{RESULTS}

This study conducted on 60 patients in two groups: group I (with gel foam) and group II (with surgical suture). With mean \pm SD of $31.12 \pm 3.29$ admitted from in the period from May 2019 to May 2020. Their gravidity ranged from 3 to 5 with median (IQR) of 4 (3-4) and their parity ranged from 2 to 4 with median (IQR) of $3(2-3)$ (Table 1).

Table (1): Demographic and characteristics of the studied patients

\begin{tabular}{|c|c|c|}
\hline \multirow{2}{*}{ Age } & Mean \pm SD & Total no. $=60$ \\
\cline { 2 - 3 } & Range & $31.12 \pm 3.29$ \\
\hline \multirow{2}{*}{ Gravity } & Median (IQR) & $27-39$ \\
\cline { 2 - 3 } & Range & $4(3-4)$ \\
\hline \multirow{2}{*}{ Parity } & Median (IQR) & $3-5$ \\
\cline { 2 - 3 } & Range & $3(2-3)$ \\
\hline
\end{tabular}


There were no cases with DM, hypertension, hepatic disease and renal

disease was included in the study (Table 2).

Table (1): Risk factors among the studied patients

\begin{tabular}{|c|c|c|}
\hline \multicolumn{2}{|c|}{} & Total no. = 60 \\
\hline DM & No & $60(100.0 \%)$ \\
\hline Hypertension & No & $60(100.0 \%)$ \\
\hline Hepatic disease & No & $60(100.0 \%)$ \\
\hline Renal disease & No & $60(100.0 \%)$ \\
\hline
\end{tabular}

There was no statistically significant difference found between group I and group II regarding age, gravity and parity of the studied cases with p-value $=0.078$, 0.463 and 0.463 respectively (Table 3 ).

Table (3): Comparison between group I (with gelfoam) and group II (with surgical suture) regarding demographic and characteristics of the studied patients

\begin{tabular}{|c|c|c|c|c|}
\hline \multirow{2}{*}{ Parameters } & Groups & Group I & Group II & \multirow{2}{*}{ P-value } \\
\cline { 3 - 4 } & Mean \pm SD & No. $=\mathbf{3 0}$ & No. $=\mathbf{3 0}$ & \\
\cline { 2 - 4 } Age & Range & $27-37 \pm 2.74$ & $31.87 \pm 3.66$ & \multirow{2}{*}{0.078} \\
\hline \multirow{2}{*}{ Gravity } & Median (IQR) & $4(3-4)$ & $27-39$ & \\
\cline { 2 - 4 } & Range & $3-5$ & $3-5$ & \multirow{2}{*}{0.463} \\
\hline \multirow{2}{*}{ Parity } & Median (IQR) & $3(2-3)$ & $3(2-3)$ & \multirow{2}{*}{0.463} \\
\cline { 2 - 4 } & Range & $2-4$ & $2-4$ & \\
\hline
\end{tabular}

$\bullet:$ Independent t-test; $\neq$ : Mann-Whitney test

Ten patients were drained in group I $(33.3 \%)$ and 9 patients were drained in group II (30.0\%) with no statistically significant difference between the two studied groups with $\mathrm{p}$-value $=0.781$. Also serous angious fluid ranged in group I between $200-400 \mathrm{mg}$ with mean \pm of 290.0 \pm 68.92 and in group II between $200-400$ with mean \pm SD of $294.44 \pm 63.66$ with no statistically significant difference between the two groups with p-value $=0.886$.
The table also showed that there was no statistically significant difference between the two groups regarding abdominal distension (10\% vs $13.3 \%$ in group I and group II respectively) with pvalue $=0.688$ and also no statistically significant difference between the two groups regarding fever (13.3\% vs $13.3 \%$ in group I and group II respectively) with $\mathrm{p}$-value $=1.000($ Table 4) . 
Table (4): Comparison between group I and group II regarding drain, serous angious fluid, abdominal distension and fever

\begin{tabular}{|c|c|c|c|c|}
\hline \multirow{2}{*}{\multicolumn{2}{|c|}{$\begin{array}{ll}\text { Parameters } & \text { Groups } \\
\end{array}$}} & Group I & Group II & \multirow{2}{*}{ P-value } \\
\hline & & No. $=30$ & No. $=30$ & \\
\hline \multirow{2}{*}{ Drain } & No & $20(66.7 \%)$ & $21(70.0 \%)$ & \multirow{2}{*}{0.781} \\
\hline & Yes & $10(33.3 \%)$ & $9(30.0 \%)$ & \\
\hline \multirow{2}{*}{ Serous angious fluid } & Mean \pm SD & $290.00 \pm 68.92$ & $294.44 \pm 63.66$ & \multirow{2}{*}{0.886} \\
\hline & Range & $200-400$ & $200-400$ & \\
\hline \multirow{2}{*}{ Abdominal distension } & No & $24(80.0 \%)$ & $29(96.7 \%)$ & \multirow{2}{*}{0.044} \\
\hline & Yes & $6(20.0 \%)$ & $1(3.3 \%)$ & \\
\hline \multirow{2}{*}{ Fever } & No & $23(76.7 \%)$ & $29(96.7 \%)$ & \multirow{2}{*}{0.023} \\
\hline & Yes & $7(23.3 \%)$ & $1(3.3 \%)$ & \\
\hline
\end{tabular}

\section{DISCUSSION}

Topical hemostaties, used in different ways as a mixture of max, grease, and barely in an effort to stop bleeding (Achneck et al., 2010). Advances in biotechnology have resulted in the development of topical hemostatic agents such agents range from absorbable topical hemostats, such as gelatin mien fibriller collagen, and regenerated oxidized cellulose to biologically active topical hemostats such as thrombin, biological adhesives (Behrens et al., 2014).

Gelatin is a hemostatis agent made from hydrolyzoel and purified animal collagen gelatins are useful as a physical matrix for clot initiation (Charlesworth et al., 2012).

The gelatin matrix is absorbed within four to six weeks case of use, low price and good hemostatic activity make topical hemostats with gelatin matrix a popular tool for reducing the morbidity caused by hemorrhage (Folić et al., 2012).

Local hemostats offer an alternative method for the hemostasis of bleedings (Chapman et al., 2010). Gelatin sponge, which is derived from porcine dermal gelatin, has been used for local hemostasis (Velyvis, 2015). By reducing intraoperative blood loss, local hemostats reduce the use of systemic hemostats, shorten the time required to achieve hemostasis, decrease the need for transfusion and its associated risks, and shorten the duration of surgical operations and hospital stay (Tomizawa, 2010).

The use of gelatin sponge may cause various adverse effects, including acute or chronic hypersensitivity reactions, granuloma formation. Moreover, gelatin sponge may act as a focus for infections.

The absorption time of gelatin sponge may vary depending on the size and location of placement, but is generally considered to be approximately 5 weeks (Charlesworth et al., 2012).

The absorption mechanism of gelatin sponge has yet to be fully elucidated. However, it has been reported that an inflammatory response is evoked around the hemostat until it is completely absorbed (Tomizawa, 2010).

The passage of first flatus is routinely used as a clinical marker for recovery of bowel motility during the postoperative period. The impairment in the recovery of bowel motility may be due to the local hypersensitivity reaction caused by the application of gelatin sponge. 
In our study, the use of gelfoam in emergency cesarean section was associated to reduce the use of systemic hemostats, shorten the time required to achieve hemostasis, decrease the need for transfusion and its associated risks, and shorten the duration of surgical operations with a prolonged time to passage of flatus and, thereby, delayed discharge from hospital.

Özer and Köstü (2017) aimed to determine the effects of use of a local hemostatic gelatin sponge (GS) on postoperative morbidity in patients undergoing cesarean section (CS). They found that use of local hemostats, shorten the time required to achieve hemostasis, decrease the need for transfusion and its associated risks, shorten the duration of surgical operations the mean time to first flatus during the postoperative period, and the duration of hospitalization are significantly prolonged by the use of gelatin sponge as a local hemostat in cesarean section patients. The delay in the recovery of bowel motility may be due to the local hypersensitivity reaction caused by gelatin sponge and/or dislocation of this local hemostat. These findings imply that the type, amount, and localization of gelatin sponges should be recorded in surgery notes. Women who experience a delay in passing the first flatus after cesarean delivery should be assessed for gelatin sponge utilization. Additionally, women who receive gelatin sponge during cesarean delivery should be monitored up closely for the recovery of postoperative intestinal motility. Further research is warranted to clarify the effects of gelatin sponge use on postoperative morbidity of women who undergo cesarean section Özer and Köstü (2017).
Ma et al. (2018) found that absorbable gelatin sponge could decrease the blood loss during hemostatic process and the postoperative drainage volume in posterior operation of lumbar degenerative disease. AGS is a safe and effective hemostatic agent in lumbar posterior surgery.

\section{CONCLUSION}

Local hemostatic agent "gel foam" is useful in controlling bleeding in cesarean section. Local hemostats reduce the use of systemic hemostats, shorten the time required to achieve hemostasis, decrease the need for transfusion and its associated risks, and shorten the duration of surgical operations and hospital stay. The delay in the recovery of bowel motility may be due to the local hypersensitivity reaction caused by gelatin sponge and/or dislocation of this local hemostat. These findings imply that the type, amount, and localization of gelatin sponges should be recorded in surgery notes.

\section{REFERENCES}

1. Abubakar II, Tillmann $\mathbf{T}$ and Banerjee A (2015): Global, regional and national age-sex specific all-cause and cause-specific mortality for 240 causes of death, 1990-2013: a systematic analysis for the Global Burden of Disease Study. Lancet, 385(9963):117-71.

2. Achneck HE, Sileshi B, Jamiolkowski RM, Albala DM, Shapiro ML and Lawson JH (2010): A comprehensive review of topical hemostatic agents: efficacy and recommendations for use. Ann Surg., 251(2):217-28.

3. Behrens AM, Sikorski MJ and Kofinas $P$ (2014): Hemostatic strategies for traumatic and surgical bleeding. Journal of Biomedical Materials Research Part A., 102(11):4182-94.

4. Chapman WC, Clavien PA and Fung J. (2010): Effective control of hepatic bleeding 
with a novel collagen-based composite combined with autologous plasma: Results of a randomized controlled trial. Arch Surg., 135:1200-4.

5. Charlesworth TM, Agthe P, Moores A and Anderson DM (2012): The use of hemostatic gelatin sponges in veterinary surgery. J Small Anim Pract., 53:51-56.

6. Folić MM, Janković SM, Folić MD and Varjačić MR. (2012): Costs of caesarean section and vaginal delivery in an uppermiddle-income country: a case series. Serbian Journal of Experimental and Clinical Research, 10(2):61-4.

7. Ma L, Dai L, Yang Y and Liu H (2018): Comparison the efficacy of hemorrhage control of Surgiflo Haemostatic Matrix and absorbable gelatin sponge in posterior lumbar surgery: a randomized controlled study. Medicine, 97(49): 136-145.

8. Ononge $S$, Mirembe $F$, Wandabwa $J$ and Campbell OM (2016): Incidence and risk factors for postpartum hemorrhage in Uganda. Reproductive Health, 13(1):38-43.

9. Oz MC, Rondinone JF and Shargill NS (2003): FloSeal Matrix: new generation topical hemostatic sealant. J Card Surg., 18(6):486-93.
10. Özer A and Köstü B (2017): Use of Gelatin Sponge Affects Postoperative Morbidity In Cesarean Section Patients. Medical Science Monitor: International Medical Journal of Experimental and Clinical Research, 23:114145.

11. Reuthebuch O, Lachat ML, Vogt $P$, Schurr $U$ and Turina $M$ (2000): FloSeal: a new hemostyptic agent in peripheral vascular surgery. Vasa., 29(3):204-6.

12. Sebghati $M$ and Chandraharan $E$ (2017): An update on the risk factors for and management of obstetric haemorrhage. Women's Health, 13(2):34-40.

13. Tomizawa Y (2010): Clinical benefits and risk analysis of topical hemostats: A review. $\mathbf{J}$ Artif Organs, 8:137-42.

14. Velyvis JH (2015): Gelatin matrix use reduces postoperative bleeding after total knee arthroplasty. Orthopedics, 38: 118-23.

15. Weeks A (2015): The prevention and treatment of postpartum haemorrhage: what do we know, and where do we go to next? BJOG: An International Journal of Obstetrics \& Gynaecology, 122(2):202-10.

16. Wormer KC and Bryant SB (2018): Pregnancy, Acute Postpartum Hemorrhage. J Obstet Gynaecol India, 69(1):31-36. 


\section{دور أدوية التجلط الموضو عية كعامل مساعد للتحكم في

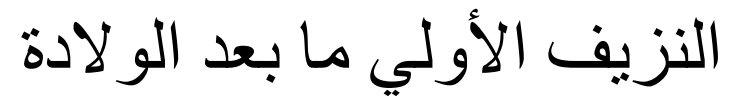

محمد الصغير الحوفي، حسام الدين حسين كامل، عادل علي البغدادي

قسم التوليد وأمراض النساء، كلية الطب، جامعة الازهر

E-mail: mohamedelhofey007@gmail.com

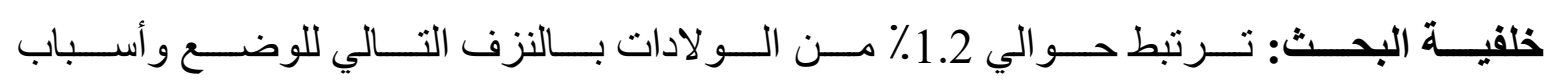

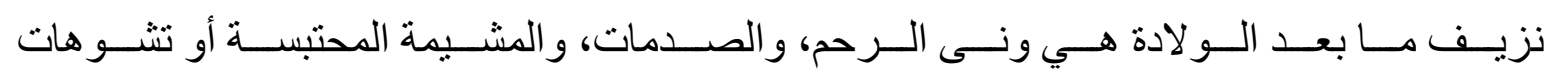
المشيمة، وتجلط الدم.

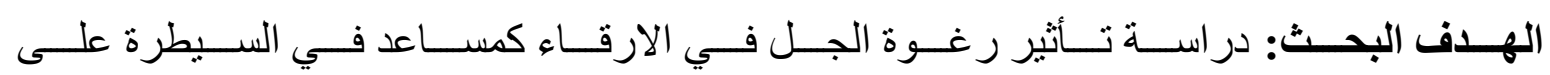
نزيف ما بعد الولادة الأولي.

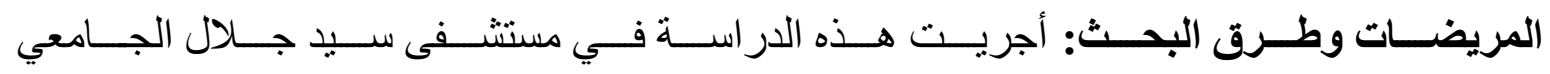

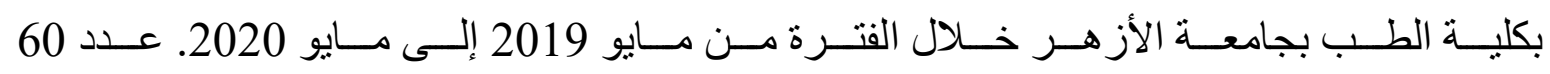

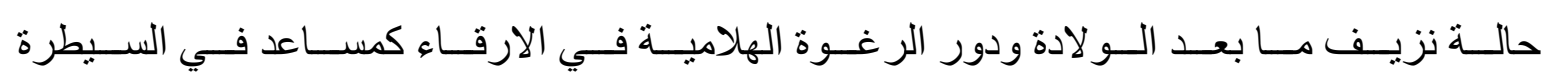
على نزيف ما بعد الو لادة الأولي.

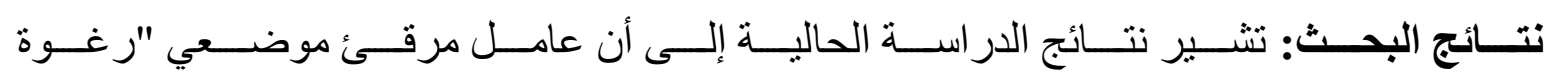

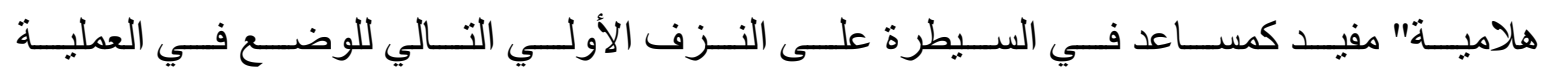

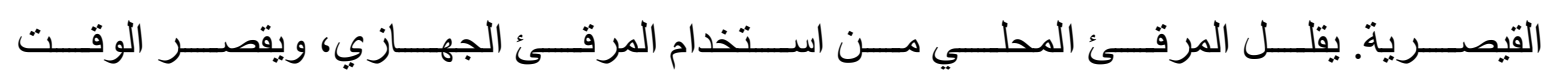

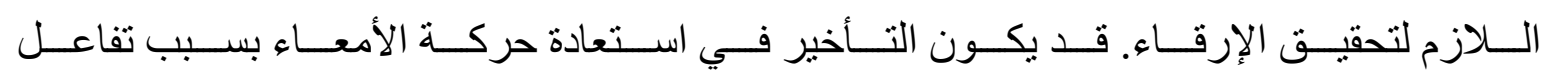

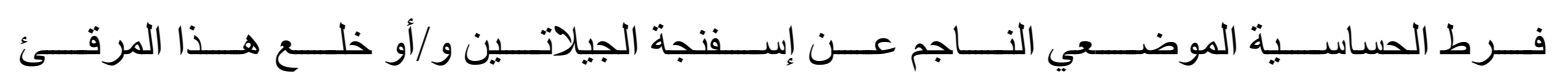
الموضعي.

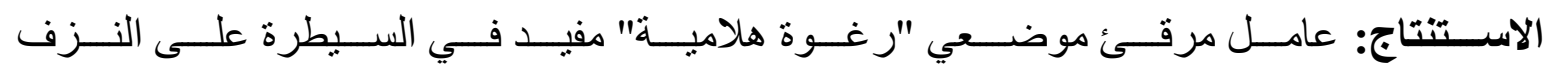
الأولي التالي للوضع في العملية القيصرية.

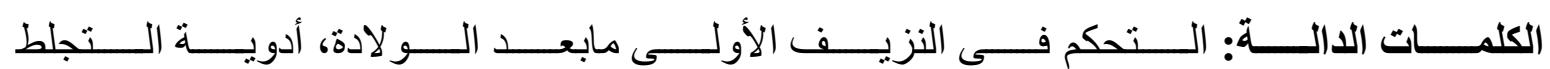
الموضو عية. 